\title{
Comparative analysis of the efficacy and accuracy of magnetic resonance imaging (MRI) and contrast-enhanced CT for residual and new lesions after transcatheter arterial chemoembolization (TACE) in patients with primary liver cancer
}

\author{
Liang Shao ${ }^{1}$, Xiaolei Wang ${ }^{1}$, Yongtao Yu ${ }^{2}$, Jiangwei Xie ${ }^{2}$ \\ ${ }^{1}$ Department of Radiology, Maternity and Child Health Care of Zaozhuang, Zaozhuang, China; ${ }^{2}$ Department of Radiology, Linyi Central Hospital, \\ Linyi, China \\ Contributions: (I) Conception and design: L Shao, X Wang; (II) Administrative support: Y Yu; (III) Provision of study materials or patients: Y Yu; \\ (IV) Collection and assembly of data: Both authors; (V) Data analysis and interpretation: L Shao, X Wang; (VI) Manuscript writing: Both authors; \\ (VII) Final approval of manuscript: Both authors. \\ Correspondence to: Yongtao Yu. Department of Radiology, Linyi Central Hospital, Linyi 276402, China. Email: 2749901187@qq.com.
}

Background: The purpose of this study was to investigate the efficacy and accuracy of magnetic resonance imaging (MRI) and contrast-enhanced computed tomography (CECT) for residual and new lesions after transcatheter arterial chemoembolization (TACE) in patients with primary liver cancer (PHC).

Methods: Seventy-two PHC patients admitted to Linyi Central Hospital from May 2018 to May 2020 were selected as the study subjects, with a total of 92 lesions. All patients were treated with TACE, and were followed up at 6 months postoperatively. In addition, all patients underwent digital subtraction angiography (DSA), and with DSA results serving as the gold standard for diagnosis, the efficacy and accuracy of MRI and CECT for residual and new lesions after TACE in PHC patients were evaluated.

Results: There were no significant differences in the specificity between the two diagnostic methods $(\mathrm{P}>0.05)$, and the diagnostic accuracy and sensitivity of MRI were markedly higher than those of CECT $(\mathrm{P}<0.05)$. The number of residual and new lesions diagnosed by MRI was notably higher than that by multislice spiral computed tomography (MSCT) $(\mathrm{P}<0.05)$, and the detection rate of residual and new lesions after TACE in PHC patients with different types of iodized oil deposition by MRI was significantly higher than that by CECT $(\mathrm{P}<0.05)$. Also, the number of postoperative tumor capsules diagnosed by MRI was considerably higher than that by CECT $(\mathrm{P}<0.05)$. There were no significant differences between the two diagnostic methods in the score of residual enhancement appearances in the arterial phase after surgery $(\mathrm{P}>0.05)$. Furthermore, there were no notable differences between the two diagnostic methods in the diagnosis of portal vein tumorous emboli and the source of blood supply to lesions after surgery $(\mathrm{P}>0.05)$.

Conclusions: The diagnostic accuracy and sensitivity of MRI for residual and new lesions after TACE in PHC patients were higher than those of CECT. However, these two diagnostic methods were similar in diagnosing portal vein tumorous emboli, the source of blood supply to lesions, and the score of residual enhancement appearances in the arterial phase after surgery.

Keywords: Magnetic resonance imaging (MRI); contrast-enhanced CT; primary liver cancer (PHC); transcatheter arterial chemoembolization (TACE); residual lesions; new lesions; efficacy evaluation; accuracy

Submitted Apr 14, 2021. Accepted for publication Aug 12, 2021.

doi: $10.21037 /$ tcr-21-831

View this article at: https://dx.doi.org/10.21037/tcr-21-831 


\section{Introduction}

Primary liver cancer (PHC) is a common hepatobiliary malignancy, and its pathogenic factors might be related to liver cirrhosis, viral hepatitis, and living environments $(1,2)$. Epidemiological investigation has shown that $\mathrm{PHC}$, as the fourth most common malignant tumor and the second leading cause of death in China, exhibited an upward trend in its incidence worldwide, with more male patients than females, seriously threatening the life safety of patients (3). The onset of PHC is relatively insidious, and its early clinical symptoms are not obvious. Thus, when patients present with typical clinical symptoms, they generally have entered the middle and late stages. Liver pains are the initial symptom of PHC patients, which mainly include persistent distending pains or dull pains. In addition, progressive muscular weakness, emaciation, and malnutrition are also common and typical symptoms of PHC patients (4-6). Transcatheter arterial chemoembolization (TACE) is an effective strategy causing ischemia and necrosis of the tumor tissues via blocking the related arteries of the tumor tissues with embolic agent. TACE has become the preferred treatment for patients with advanced PHC, where the appropriate amount of embolic agents are injected at an appropriate speed after selective insertion of a catheter into the target artery supplying blood to liver tumors, resulting in the ischemic necrosis of tumor tissues, so as to achieve a therapeutic effect (7). However, most patients' blood supply at tumor sites changes after treatment, and some residual tumor cells might appear in the lesions, which facilitates the recurrence of PHC. Therefore, effective imaging diagnosis for patients after TACE is of positive significance to reduce the recurrence rate of tumors and improve prognosis (8).

Clinical practice has demonstrated that contrastenhanced computed tomography (CECT) and magnetic resonance imaging (MRI) have not only provided significant advantages for the clinical differentiation of benign and malignant tumors, but have also exhibited unique superiority in the evaluation of residual and new lesions after TACE $(9,10)$. Based on this, in order to further explore the efficacy and accuracy of MRI and CECT for residual and new lesions after TACE in PHC patients, 72 PHC patients admitted to Linyi Central Hospital from May 2018 to May 2020 were selected as the study subjects, and the summary report is as follows. We present the following article in accordance with the STARD reporting checklist (available at http://dx.doi.org/10.21037/tcr-21-831).

\section{Methods}

\section{General information}

Seventy-two PHC patients admitted to Linyi Central Hospital from May 2018 to May 2020 were selected as the study subjects (Figure 1). There were 38 males and 34 females, with an average age of $52.44 \pm 3.28$ years old, and an average tumor diameter of $2.18 \pm 0.73 \mathrm{~cm}$. Among them, there were 41 patients with massive hepatocellular carcinomas and 31 patients with nodular liver cancers. Additionally, there were 25 patients in Tumor Node Metastasis (TNM) stage I, 35 patients in stage II, and 12 patients in stage III. There were also 39 patients in Child-Pugh grade A, 33 patients in grade B, and no patients in grade C. All procedures performed in this study involving human participants were in accordance with the Declaration of Helsinki (as revised in 2013).

\section{Inclusion criteria}

The inclusion criteria were as follows: (I) patients meeting the diagnostic criteria for PHC; (II) patients with no contraindications to digital subtraction angiography (DSA), CT, or MRI scanning; and (III) patients who received TACE treatment and achieved the expected survival of more than 3 months. This study was approved by the ethics committee of Linyi Central Hospital (No. 20180430), and the patients and their families were informed of the purpose and process of the study and signed the informed consent.

\section{Exclusion criteria}

The exclusion criteria were as follows: (I) patients with other malignant tumors; (II) patients with abnormal systemic coagulation or a history of gastrointestinal bleeding; (III) patients with obvious tumor thrombus in the portal veins; and (IV) patients with cognitive impairment, such as mental disorders, or those who refused to cooperate with the study.

\section{Study methods}

Patients were followed up at 6 months postoperatively, and DSA, CECT, and MRI examinations were performed in turn.

\section{DSA examinationPage5/}

After the test table was disinfected and covered with towels, 


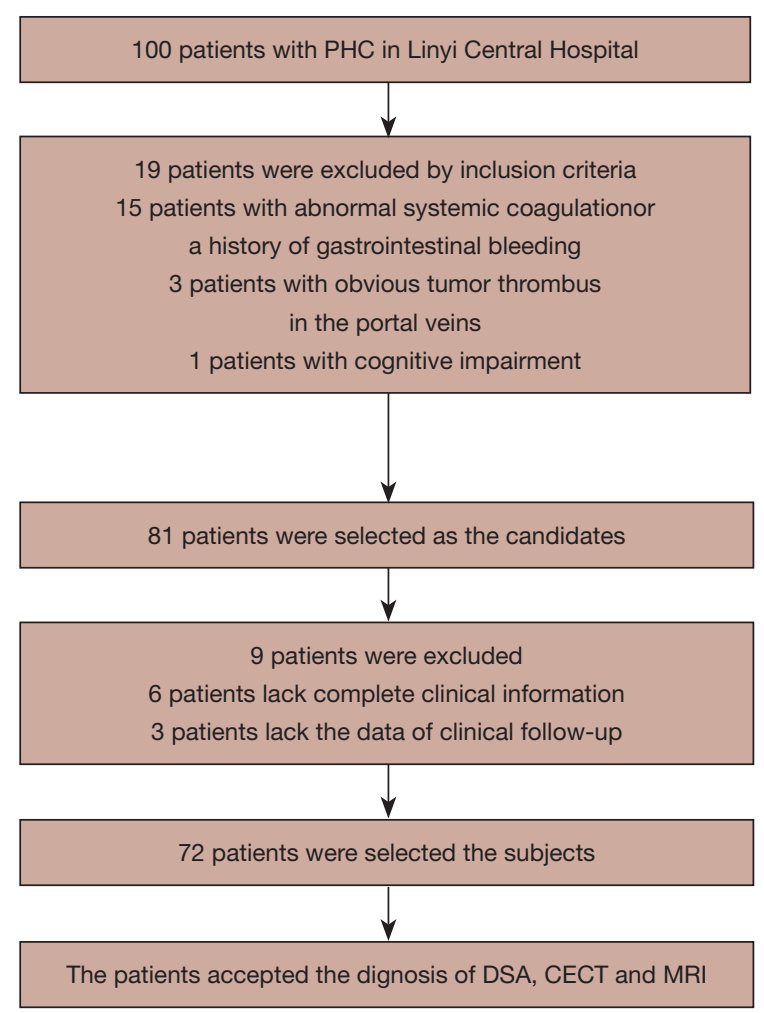

Figure 1 The flow diagram of subject selection.

patients, who were in the supine position, received femoral artery puncture after local anesthesia. After intubation, extrahepatic or hepatic arteriography was performed (11).

\section{CECT examination}

The Definition AS spiral CT scanner with 64 detector rows and 128 slices (Siemens, Germany) was utilized. Patients assumed supine positions and lay in the middle of the bed, with their arms lifted and their hands placed behind their heads. Next, transverse axial scanning was carried out, with a scanning interval of $5-10 \mathrm{~mm}$ and a slice thickness of $5-10 \mathrm{~mm}$. Ionic or non-ionic iodinated contrast agents with $55-75 \mathrm{~mL}$ of contrast material were used and the intravenous bolus injection was administered with a pressure syringe at a rate of $2.0-3.0 \mathrm{~mL} / \mathrm{s}$. Continuous scanning in the arterial phase was performed after injection, with the scanning started at $30 \mathrm{~s}-2 \mathrm{~min}$ in the portal vein phase and at $2-5 \mathrm{~min}$ in the nephrographic phase.

\section{MRI examination}

Patients in the supine position were scanned using a 9D MRI detector (Tangshan Ruijian Technology Co., Ltd.,
Hebei, China). The patients' livers were scanned in the $T_{1} W I$ sagittal plane and the $T_{2} W I$ transverse axial plane, and then the results of imaging examination were analyzed. Some patients, who were in coronary positions, were scanned in the sequence of FSE- $T_{1} W I$ in enhancement scanning (12).

\section{Evaluation indexes}

Accuracy $=$ accurate diagnosis/total number of cases $\times$ $100 \%$; sensitivity $=$ true positive $/($ true positive + false negative $) \times 100 \%$; specificity $=$ true negative $/$ true negative + false positive) $\times 100 \%$.

\section{Residual lesions}

Within 1 week postoperatively, patients underwent MRI or CECT examination, with a small amount of shadow of cancer cell lesions and residual lesions.

\section{New lesions}

At 6 months postoperatively, patients underwent MRI or CECT examination, and intrahepatic infiltration was observed, with local recurrence.

The detection of different types of iodized oil deposition in PHC patients after surgery by two diagnostic methods was recorded. The types of iodized oil deposition could be divided into the following groups: type I, with uniform iodized oil deposition in tumors; type II, with iodized oil deposition at the edge of tumors and uneven density; type III, with dispersive iodized oil deposition and a cumulative deposition area of more than $30 \%$; and type IV, with less or no iodized oil deposition and a deposition area of less than $30 \%$.

The diagnosis of tumor capsules postoperatively by two diagnostic methods was also recorded. The region of interest in the arterial phase was scored from 1 to 5 points, with no residual enhancement area scored 1 point, and residual enhancement area scored 5 points.

The DSA examination results were used as the gold standard to compare the accuracy between the two examination methods for the diagnosis of portal vein tumorous emboli and the source of blood supply to the lesions after surgery.

\section{Statistical analysis}

The data were statistically analyzed and processed by SPSS21.0 software (IBM, Armonk, NY, USA). GraphPad 
Table 1 Comparison of clinical value between the two diagnostic methods [n (\%)]

\begin{tabular}{lccc}
\hline Diagnostic method & Specificity & Accuracy & Sensitivity \\
\hline CECT & 100 & 81.58 & 73.49 \\
MRI & 100 & 96.73 & 93.46 \\
$\chi^{2}$ & 0.000 & 11.869 & 14.455 \\
$P$ & 1.000 & 0.001 & 0.000 \\
\hline
\end{tabular}

CECT, contrast-enhanced computed tomography; MRI, magnetic resonance imaging.

Table 2 Comparison of the diagnostic results of residual and new lesions after surgery [n (\%)]

\begin{tabular}{lcc}
\hline Diagnostic method & Residual lesions & New lesions \\
\hline DSA & $43(46.74 \%)$ & $49(53.26 \%)$ \\
CECT & $29(31.52 \%)$ & $38(41.30 \%)$ \\
MRI & $37(40.22 \%)$ & $46(50.00 \%)$ \\
$\chi^{2}$ & 4.170 & 5.333 \\
P & 0.041 & 0.021
\end{tabular}

CECT, contrast-enhanced computed tomography; MRI, magnetic resonance imaging.

Table 3 Comparison of detection rate of residual and new lesions after TACE in PHC patients with different types of iodized oil deposition between the two diagnostic methods [n]

\begin{tabular}{|c|c|c|c|c|c|c|c|c|c|}
\hline $\begin{array}{l}\text { Diagnostic } \\
\text { method }\end{array}$ & \multicolumn{2}{|c|}{ Type I $(\mathrm{n}=26)$} & \multicolumn{2}{|c|}{ Type II (n=34) } & \multicolumn{2}{|c|}{ Type III (n=23) } & \multicolumn{2}{|c|}{ Type IV $(n=9)$} & Total detection rate \\
\hline CECT & 10 & 16 & 21 & 13 & 15 & 8 & 9 & 0 & $73.4 \%$ \\
\hline MRI & 12 & 14 & 25 & 9 & 18 & 5 & 9 & 0 & $91.2 \% *$ \\
\hline
\end{tabular}

* means the comparison with CET, P<0.05. TACE, transcatheter arterial chemoembolization; PHC, primary liver cancer; CECT, contrastenhanced computed tomography; MRI, magnetic resonance imaging.

Prism 7 (GraphPad Software, San Diego, USA) was used to draw pictures of the data. Measurement data were expressed by $(\bar{x} \pm s)$ and tested by $t$-test. Enumeration data were expressed as $[\mathrm{n}(\%)]$ and tested by $\chi^{2}$ test. Differences were statistically significant when $\mathrm{P}<0.05$.

\section{Results}

\section{Comparison of clinical value between the two diagnostic methods}

There were no significant differences in specificity between the two diagnostic methods $(\mathrm{P}>0.05)$. However, the accuracy and sensitivity of MRI were markedly higher than those of CECT $(\mathrm{P}<0.05)$, as shown in Table 1.
Comparison of the diagnostic results of residual and new lesions after surgery

The number of residual and new lesions diagnosed by MRI was significantly higher than that by multislice spiral computed tomography (MSCT) $(\mathrm{P}<0.05)$, as shown in Table 2.

Comparison of detection rate of residual and new lesions after TACE in PHC patients with different types of iodized oil deposition between the two diagnostic methods

The detection rate of residual and new lesions after TACE in PHC patients with different types of iodized oil deposition diagnosed by MRI was significantly higher than that by CECT $(\mathrm{P}<0.05)$, as shown in Table 3. 


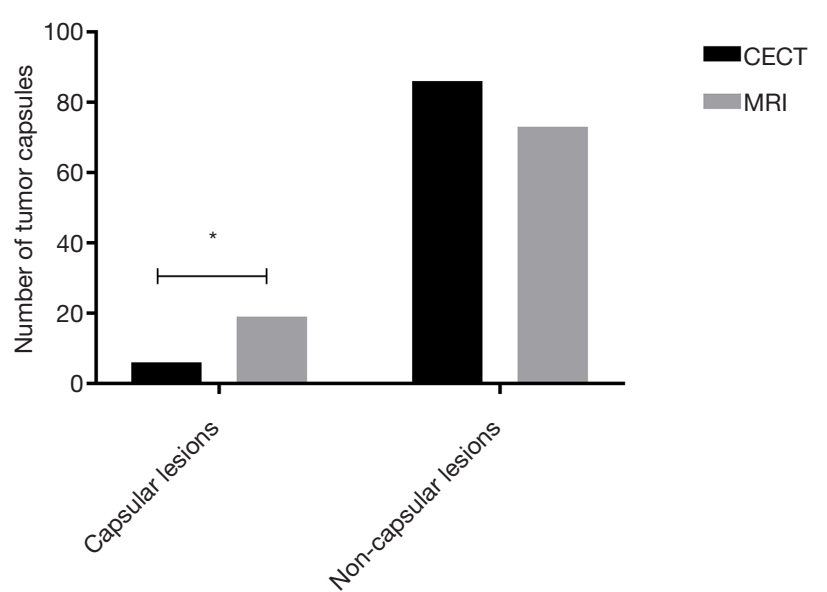

Figure 2 Comparison of diagnosis of tumor capsules after surgery between the two diagnostic methods [n (\%)]. The abscissa indicates capsular lesions and non-capsular lesions, while the ordinate indicates the number of tumor capsules. There were six $(6.52 \%)$ capsular lesions and 86 (93.48\%) non-capsular lesions diagnosed by CECT, and 19 (20.65\%) capsular lesions and 73 (79.35\%) noncapsular lesions diagnosed by MRI. * indicates that there were significant differences in the number of tumor capsules diagnosed by the two methods $\left(x^{2}=7.823, \mathrm{P}=0.005\right)$. CECT, contrast-enhanced computed tomography; MRI, magnetic resonance imaging.

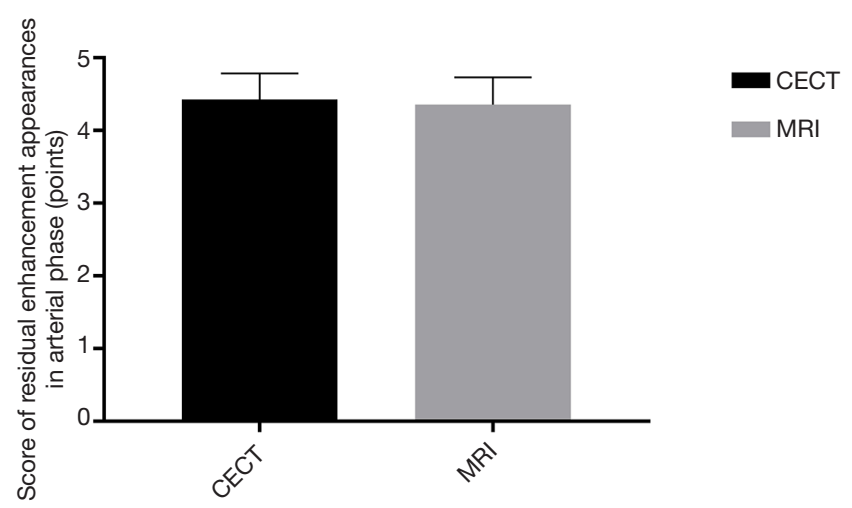

Figure 3 Comparison of residual enhancement appearances in arterial phase between the two diagnostic methods $(\bar{x} \pm s)$. The abscissa indicates CECT and MRI, while the ordinate indicates the score of residual enhancement appearances in the arterial phase. The score of residual enhancement appearances in the arterial phase diagnosed by CECT was $4.17 \pm 0.51$ points, while that diagnosed by MRI was $4.09 \pm 0.53$ points. CECT, contrastenhanced computed tomography; MRI, magnetic resonance imaging.

\section{Comparison of diagnosis of tumor capsules after surgery} between the two diagnostic methods

The number of tumor capsules in patients diagnosed by MRI was significantly higher than that by CECT $(\mathrm{P}<0.05)$, as shown in Figure 2.

\section{Comparison of residual enhancement appearances in the arterial phase between the two diagnostic methods}

There were no significant differences between the two diagnostic methods in the score of residual enhancement appearances in the arterial phase after surgery $(\mathrm{P}>0.05)$, as shown in Figure 3.

\section{Comparison of portal vein tumorous emboli and the source of blood supply to lesions between the two diagnostic methods}

There was no significant difference between the two diagnostic methods in the diagnosis of portal vein tumorous emboli and the source of blood supply to lesions after surgery ( $\mathrm{P}>0.05)$, as shown in Table 4.

\section{Imaging comparison of patients with different diagnostic methods before and after surgery}

The imaging comparison of patients with different diagnostic methods before and after surgery is shown in Figure $4 A-4 D$.

\section{Discussion}

TACE, as a common method for the treatment of PHC, changes the growth environment of tumor cells by using a high concentration of chemotherapeutics to promote the coagulation and necrosis of tumor cells, which can effectively inhibit the growth of liver cancer cells, reduce cancer cell metastasis, check the progress of the disease, and improve the prognosis of patients (13-15). However, due to the complex structure of liver tissues and multiple blood supply mechanisms in tumor tissues, TACE cannot completely block the blood supply to tumors and will promote the occurrence of residual lesions, providing an anatomical basis for new lesions (16). Therefore, early evaluation of tumor activity in PHC patients after TACE is crucial in order to formulate clinical treatment plans that 
Table 4 Comparison of portal vein tumorous emboli and the source of blood supply to lesions between the two diagnostic methods after surgery [n $(\%)]$

\begin{tabular}{|c|c|c|c|c|}
\hline Diagnostic method & \multicolumn{2}{|c|}{ Source of blood supply to the lesions } & \multicolumn{2}{|c|}{ Portal vein tumorous emboli } \\
\hline CECT & 69 & 23 & 17 & 75 \\
\hline MRI & 73 & 19 & 14 & 78 \\
\hline$\chi^{2}$ & \multicolumn{2}{|c|}{0.494} & \multicolumn{2}{|c|}{0.349} \\
\hline
\end{tabular}

CECT, contrast-enhanced computed tomography; MRI, magnetic resonance imaging.
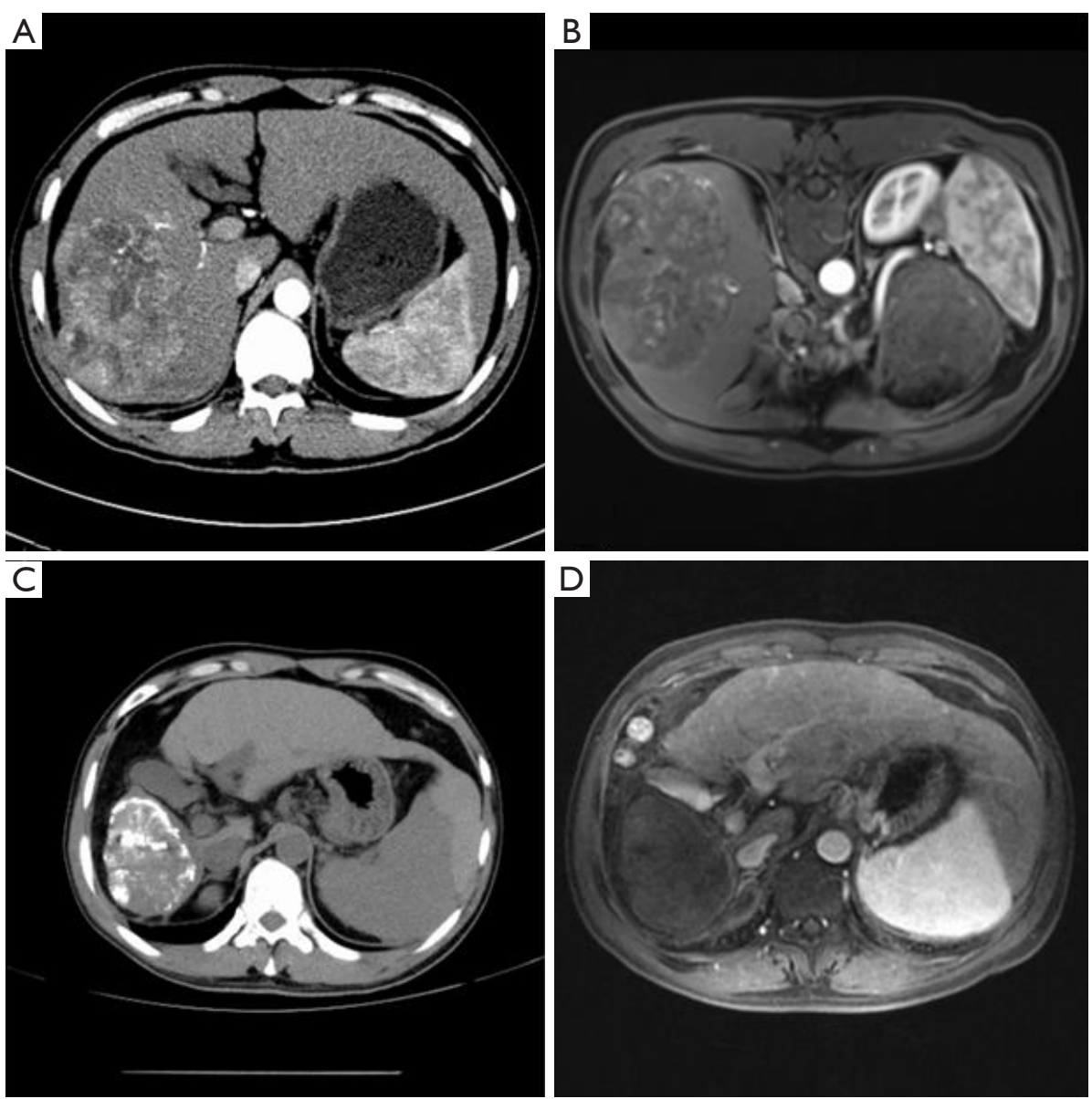

Figure 4 Imaging comparison of patients with different diagnostic methods before and after surgery. A shows a preoperative CECT image; B shows a preoperative MRI image; C displays a postoperative MRI image; D displays a postoperative CECT image. CECT, contrastenhanced computed tomography; MRI, magnetic resonance imaging.

improve prognosis and prolong the survival of patients. At present, DSA, CECT, and MRI are the most common methods for determining the postoperative efficacy of PHC patients. Among them, CECT is mainly used for the clinical examination of hepatic artery fistula and tumor nutrient vessels, and the contrast agents used in CECT easily interfere with the clear display of tumor tissues during scanning. MRI, which provides advantages such as stereo 
imaging and high-definition imaging in clinical application, can clearly show liver lesions and the anatomical relationship of surrounding tissues, providing the basis for formulating clinical treatment plans for patients (17-19).

In this study, the DSA results were taken as the standard, and the efficacy and accuracy of CECT and MRI in examining residual and new lesions after surgery were analyzed and compared. The results showed that the accuracy and sensitivity of residual and new lesions in PHC patients detected by MRI was significantly higher than that by CECT $(\mathrm{P}<0.05)$. Mapelli et al. $(20)$ pointed out that the accuracy and sensitivity of CECT were $82.31 \%$ and $71.96 \%$, respectively, which were significantly lower than the $93.68 \%$ and $93.29 \%$ of MRI, respectively. This demonstrated that MRI examination is more comprehensive and accurate in evaluating the activity of tumor lesions in patients postoperatively, and its accuracy and sensitivity in detecting residual and new lesions in patients after surgery were also higher. As shown in Figure 4, the MRI imaging diagnosis results of patients were clearer than those of CECT before and after surgery, and MRI could clearly show the residual and new lesions both pre- and postoperatively, thereby providing a basis for the formulation of subsequent treatment plans.

Clinical studies have found that the tumor capsules around the liver lesions of PHC patients often appear due to embolism, and have a particular defensiveness, and can inhibit the growth and metastasis of tumor cells to a certain extent. Therefore, the clinical diagnosis of the liver tumor capsules in PHC patients has a positive significance for the diagnosis of the lesions after TACE (21). The present study demonstrated that the number of tumor capsules detected by MRI was significantly higher than that by CECT $(\mathrm{P}<0.05)$, illustrating that MRI could clearly show the conditions of patients' tumor capsules after surgery. In addition, studies have confirmed that MRI can make multi-plane presentation, clearly showing patients' organs and lesions. Moreover, it is combined with a flowing void effect, greatly improving the efficacy of clinical diagnosis. By comparing different types of iodized oil deposition in patients, we found that there were marked differences in clinical prognosis. Patients with a large area and uniform distribution of iodized oil deposition had a lower incidence of new lesions and better prognosis after surgery $(22,23)$. Moreover, TACE is more effective than conventional chemotherapy in advance PHC treatment compared, which could effectively improve the tumor focuses of the patients. However, some adverse events such as the high toxicity on liver limit the application of TACE in PHC treatment compared with conventional chemotherapy. This study confirmed that the detection rate of MRI in patients with different types of iodized oil deposition was higher than that of TACE, suggesting that MRI exhibited notable advantages in the diagnosis of tumor status in PHC patients after surgery.

In conclusion, the accuracy and sensitivity of MRI in the diagnosis of residual and new lesions after TACE in PHC patients were significantly higher than those of CECT. However, there were no significant differences between the two diagnostic methods in portal vein tumorous emboli, the source of blood supply to lesions, and the score of enhancement appearances in the arterial phase.

\section{Acknowledgments}

Funding: None.

\section{Footnote}

Reporting Checklist: The authors have completed the STARD reporting checklist. Available at http://dx.doi.org/10.21037/ tcr-21-831

Data Sharing Statement: Available at http://dx.doi. org/10.21037/tcr-21-831

Conflicts of Interest: Both authors have completed the ICMJE uniform disclosure form (available at http://dx.doi. org/10.21037/tcr-21-831). Both authors have no conflicts of interest to declare.

Ethical Statement: The authors are accountable for all aspects of the work in ensuring that questions related to the accuracy or integrity of any part of the work are appropriately investigated and resolved. All procedures performed in this study involving human participants were in accordance with the Declaration of Helsinki (as revised in 2013). This study was approved by the ethics committee of Linyi Central Hospital (No. 20180430), and the patients and their families were informed of the purpose and process of the study and signed the informed consent. The trial was registered in Chinese Clinical Trial Registry (ChiCTR2100048819).

Open Access Statement: This is an Open Access article distributed in accordance with the Creative Commons 
Attribution-NonCommercial-NoDerivs 4.0 International License (CC BY-NC-ND 4.0), which permits the noncommercial replication and distribution of the article with the strict proviso that no changes or edits are made and the original work is properly cited (including links to both the formal publication through the relevant DOI and the license). See: https://creativecommons.org/licenses/by-nc-nd/4.0/.

\section{References}

1. Wang G, Bi C. Correlations of pri-Let-7 gene polymorphisms with the recurrence and metastasis of primary liver cancer after transcatheter arterial chemoembolization. Pathol Res Pract 2018;214:667-72.

2. Schaub SK, Apisarnthanarax S, Price RG, et al. Functional Liver Imaging and Dosimetry to Predict Hepatotoxicity Risk in Cirrhotic Patients With Primary Liver Cancer. Int J Radiat Oncol Biol Phys 2018;102:1339-48.

3. Chen PY, Fang AP, Wang XY, et al. Adherence to the Chinese or American Dietary Guidelines is Associated with a Lower Risk of Primary Liver Cancer in China: A Case-Control Study. Nutrients 2018;10:1113.

4. Nautsch F, Ludwig JM, Xing M, et al. Racial Disparities and Sociodemographic Differences in Incidence and Survival Among Pediatric Patients in the United States With Primary Liver Cancer: A Surveillance, Epidemiology, and End Results (SEER) Population Study. J Clin Gastroenterol 2018;52:262-7.

5. Fujita M, Yamaguchi R, Hasegawa T, et al. Classification of primary liver cancer with immunosuppression mechanisms and correlation with genomic alterations. EBioMedicine 2020;53:102659.

6. Jeong $\mathrm{S}$, Zheng B, Wang $\mathrm{H}$, et al. Nervous system and primary liver cancer. Biochim Biophys Acta Rev Cancer 2018;1869:286-92.

7. Liao CK, Chiang JM, Tsai WS, et al. Primary tumor location in stage III colon cancer has prognostic impact on subsequent liver metastasis. J Surg Oncol 2018;118:1301-10.

8. Engel LS, Zabor EC, Satagopan J, et al. Prediagnostic serum organochlorine insecticide concentrations and primary liver cancer: A case-control study nested within two prospective cohorts. Int J Cancer 2019;145:2360-71.

9. Prayongrat A, Kobashi K, Ito YM, et al. The normal tissue complication probability model-based approach considering uncertainties for the selective use of radiation modality in primary liver cancer patients. Radiother Oncol 2019;135:100-6.
10. Tran KT, Coleman HG, McCain RS, et al. Serum Biomarkers of Iron Status and Risk of Primary Liver Cancer: A Systematic Review and Meta-Analysis. Nutr Cancer 2019;71:1365-73.

11. Opstelten JL, Chan SSM, Hart AR, et al. Prediagnostic Serum Vitamin D Levels and the Risk of Crohn's Disease and Ulcerative Colitis in European Populations: A Nested Case-Control Study. Inflamm Bowel Dis 2018;24:633-40.

12. Chen X, Chang Z, Liu Z. D-dimer increase: an unfavorable factor for patients with primary liver cancer treated with TACE. Cancer Chemother Pharmacol 2019;83:797-802.

13. Wen L, Xin B, Wu P, et al. An Efficient Combination Immunotherapy for Primary Liver Cancer by Harmonized Activation of Innate and Adaptive Immunity in Mice. Hepatology 2019;69:2518-32.

14. Matsuyama TA, Ohuchi H, Saito K, et al. Occurrence of a primary liver cancer with an unusual histologic appearance as a late Fontan complication. Pathol Res Pract 2019;215:152366.

15. Zhao Y, Yang J, Luo M, et al. Contrast-Enhanced CTbased Textural Parameters as Potential Prognostic Factors of Survival for Colorectal Cancer Patients Receiving Targeted Therapy. Mol Imaging Biol 2021;23:427-35.

16. Gettys FK, Carpenter A, Stasikelis PJ. The Role of MRI in Children With Congenital Limb Deficiencies With Associated Scoliosis. J Pediatr Orthop 2020;40:e390-3.

17. Xu X, Zhang HL, Liu QP, et al. Radiomic analysis of contrast-enhanced CT predicts microvascular invasion and outcome in hepatocellular carcinoma. J Hepatol 2019;70:1133-44.

18. Pinkham DW, Negahdar M, Yamamoto T, et al. A Feasibility Study of Single-inhalation, Single-energy Xenon-enhanced CT for High-resolution Imaging of Regional Lung Ventilation in Humans. Acad Radiol 2019;26:38-49.

19. Tavazzi E, Zivadinov R, Dwyer MG, et al. MRI biomarkers of disease progression and conversion to secondaryprogressive multiple sclerosis. Expert Rev Neurother 2020;20:821-34.

20. Mapelli P, Ironi G, Bergamini A, et al. Synergic role of preoperative 18F-fluorodeoxyglucose PET and MRI parameters in predicting histopathological features of endometrial cancer. Nucl Med Commun 2020;41:1073-80.

21. Wong WD, Mohammed MF, Nicolaou S, et al. Impact of Dual-Energy CT in the Emergency Department: Increased Radiologist Confidence, Reduced Need for Follow-Up Imaging, and Projected Cost Benefit. AJR Am 
J Roentgenol 2020;215:1528-38.

22. Piccardo A, Trimboli P, Rutigliani M, et al. Additional value of integrated (18)F-choline PET/4D contrastenhanced CT in the localization of hyperfunctioning parathyroid glands and correlation with molecular profile. Eur J Nucl Med Mol Imaging 2019;46:766-75.

Cite this article as: Shao L, Wang X, Yu Y, Xie J. Comparative analysis of the efficacy and accuracy of magnetic resonance imaging (MRI) and contrast-enhanced CT for residual and new lesions after transcatheter arterial chemoembolization (TACE) in patients with primary liver cancer. Transl Cancer Res 2021;10(8):3739-3747. doi: 10.21037/tcr-21-831
23. Marie-Hardy L, Barut N, Sari Ali H, et al. Evaluation of disc degeneration adjacent to AOspine A fractures: preand post-operative MRI analysis. SICOT J 2020;6:33.

(English Language Editor: A. Kassem) 